\title{
Análise dos fatores de risco anteparto para ocorrência de cesárea
}

\author{
Analysis of the risk factors for cesarean section \\ Simone Angélica Leite de Carvalho Silva ${ }^{1}$, Olimpio Barbosa de Moraes Filho ${ }^{2}$, \\ Cícero Ferreira Fernandes Costa ${ }^{3}$
}

\section{RESUMO}

Objetivos: criar um modelo preditivo para ocorrência de cesárea na Maternidade Professor Monteiro de Morais após avaliação dos fatores de risco anteparto das gestantes que pariram no período de 1 de setembro de 1999 a 31 de agosto de 2000, e posteriormente verificar a eficiência do serviço na indicação de cesárea. Métodos: foi realizado estudo longitudinal, do tipo caso-controle com 3626 gestantes, no qual se verificou quais os fatores anteparto foram considerados de risco para cesárea, no período de 1 de setembro de 1999 a 31 de agosto de 2000. Posteriormente, criou-se modelo preditivo ideal, o qual permitiu quantificar o risco de cesárea para cada paciente na presença de um ou mais fatores de risco. A seguir, aplicou-se o modelo à amostra do estudo a fim de verificar o grau de concordância entre o risco previsto de cesárea e a realização do ato cirúrgico, ou seja, a eficácia na indicação de cesariana. Resultados: aplicando-se o modelo preditivo na amostra, verificou-se que na ausência dos fatores de riscos anteparto, o risco basal de cesárea foi de 15,2\%. O grau de concordância entre o previsto pelo modelo logístico e a ocorrência de cesárea foi de $86,6 \%$. Conclusões: o modelo logístico permitiu identificar o risco basal de cesárea e quantificar a probabilidade de cesárea a partir da introdução do fator de risco. O modelo pode ser considerado útil e eficaz, uma vez que houve concordância entre o acerto e o previsto em $86,6 \%$ para cesariana, e $53,6 \%$ das pacientes submetidas ao parto normal, de fato, não tinham fator de risco para cesárea.

PALAVRAS-CHAVE: Cesárea; Resolução de problemas; Parto normal; Fatores de risco

\section{ABSTRACT}

Purpose: to create a predictive model for cesarean section at the "Professor Monteiro de Morais Maternity" after evaluation of antepartum risk factors of the pregnant women who delivered from September 1, 1999 to August 31, 2000, and then, to verify the efficacy of indication for cesarean section. Methods: a longitudinal, case control study with 3.626 pregnant women was performed to identify the antepartum risk factors for cesarean section in the period from September 1, 1999 to August 31, 2000. Thereafter an ideal model able to quantify the risk for cesarean section for each patient in the presence of one or more risk factor was created. Then, the model was applied to the patients of the study in order to verify the efficacy of indication for cesarean section. Results: the baseline risk for cesarean section was $15.2 \%$. The concordance between the percentage estimated through logistic model and cesarean delivery was $86.6 \%$. Conclusions: the logistic model was able to identify the baseline risk for cesarean section and to quantify the increase in risk for cesarean section in each patient when risk factors were introduced in the model. The model can be considered efficient and able to predict cesarean section because the agreemant between the prediction and the correct indication was $86.6 \%$, and $53.6 \%$ of the patients who had vaginal delivery did not have any risk factor for cesarean section.

KEYWORDS: Cesarean section; Cesarean delivery; Predictive model; Natural childbirth; Risk factors; Problem salving

\section{Introdução}

A operação cesariana é a tocurgia cujas taxas vêm aumentando significativamente no mundo e no Brasil $^{1,2}$. Essa ascensão fez com que a cesárea fosse considerada epidêmica pelas autoridades de saúde ${ }^{3}$. Existe muita controvérsia sobre qual seria o índice aceitável de cesariana em um serviço de obste-

1 Médica Assistente, Preceptora da Maternidade da Encruzilhada-CISAM, da Universidade de Pernambuco - (PE)

2 Professor da Disciplina de Ginecologia e Obstetricia da Faculdade de Ciências Médicas da Universidade de Pernambuco - (PE)

3 Professor Livre-docente da Disciplina de Ginecologia e Obstetrícia da Faculdade de Ciências Médicas da Universidade de Pernambuco - (PE)

Correspondência: Simone Angélica Leite de Carvalho Silva

Rua Capitão Sampaio Xavier, 429 apto. 1801 - Rosarinho - 52050-210 - Recife - PE - Fone: (81) 3242-5276 - e-mail: carvalho.sa@pop.com.br 
trícia ${ }^{4}$. Há consenso de que as taxas atuais de cesariana são muitos elevadas e que se deve buscar reduzi-las a niveis mais aceitáveis, sem prejudicar, com essa conduta, a qualidade de vida dos recém-nascidos ${ }^{5}$.

Fatores institucionais, sociais e legais estão associados com o aumento da incidência de cesárea ${ }^{6}$. O fato de o obstetra ser o principal provedor a cuidar da gestante, a expectativa da mesma em ter um tratamento personalizado e o horário confuso dos obstetras são alguns dos motivos para a marcação de cesáreas eletivas. Outro motivo é o receio do médico em realizar parto vaginal em gestantes com cesárea anterior ${ }^{7}$.

Vários outros fatores de risco para cesárea têm sido estudados, incluindo parturição na adolescência ${ }^{5}$, idade maior ou igual a 35 anos no primeiro parto ${ }^{8}$, nuliparidade ${ }^{9}$ e gestação pós-termo ${ }^{10}$.

Algumas intercorrências obstétricas têm contribuído para o aumento da taxa de cesárea. Dentre elas, destacam-se: sindrome hipertensiva, síndrome hemorrágica no $3^{\circ}$ trimestre gestacional, ruptura prematura das membranas, gemelaridade, diabete gestacional e apresentação pélvica ${ }^{5}$.

Não existem dúvidas sobre a importância da cesárea como uma técnica apropriada para o manejo de uma série de situações obstétricas específicas, que necessitam da interrupção da gestação como a única maneira de preservar a saúde da mulher ou do feto. Este recurso de efeito benéfico tão claro não é, todavia, ainda hoje acessivel a uma grande parcela da população de mulheres das regiões menos desenvolvidas. Por outro lado, já existem evidências suficientes que permitem dizer que não ocorre diminuição sistemática e contínua da morbidade e mortalidade perinatais com o aumento da taxa de cesárea. A falsa associação de causa-efeito entre o aumento da taxa de cesárea e a diminuição da mortalidade perinatal foi responsável por um respaldo pseudocientífico para um aumento indiscriminado da prática de cesarianas em todo o mundo ocidental e particularmente no Brasil ${ }^{3}$.

O desejo de reverter o aumento nas taxas de cesariana e tentar situá-las em niveis mais próximos daqueles que se podem considerar adequados exige revisão das condutas naquelas indicações que mais contribuíram para que a incidência dos partos operatórios atingisse os valores registrados ${ }^{6}$. Outra estratégia é analisar a tendência da incidência de cesárea de acordo com os fatores de riscos clínicos e não clínicos para cesárea ${ }^{11}$. Desta forma, pode-se determinar o impacto das mudanças na distribuição dos fatores de risco e daquelas na prática médica para cada nível de risco ${ }^{11}$.
Por último, a criação de um modelo preditivo para operação cesariana, que permita quantificar o risco de cesárea, poderá ajudar a decisão no modo pelo qual ocorrerá o parto. Desta forma, haverá a possibilidade de redução da incidência da cesariana, sem comprometimento materno-fetal. Esta tendência e preocupação já são antigas, como mostra o trabalho realizado por Hin et al., em $1997^{12}$, e se perpetuam até o presente momento, como demonstraram, recentemente, Cunha et al. em estudo publicado em $2002^{5}$.

O objetivo deste trabalho foi criar um modelo preditivo para ocorrência de cesárea na Maternidade Professor Monteiro de Morais após avaliação de fatores de risco anteparto e posteriormente verificar a eficiência do serviço na indicação de cesárea.

\section{Métodos}

O presente estudo foi do tipo longitudinal, caso-controle, uma vez que, a partir da constatação da via de parto, foram formados os grupos: cesárea (grupo caso) e parto vaginal (grupo controle), para análise da influência dos fatores anteparto sobre o episódio da cesárea.

Participaram do estudo gestantes que pariram recém-nascidos vivos por cesárea ou parto vaginal no período de 1 de setembro de 1999 a 31 de agosto de 2000, na Maternidade Professor Monteiro de Morais - CISAM, Recife, PE.

Os critérios de inclusão das parturientes ao estudo foram nascimento de recém-nascidos com peso mínimo de 1.000 g e apresentação cefálica. Os seguintes critérios de exclusão foram também considerados: ausência do registro de diagnóstico de admissão na ficha obstétrica, antecedente de duas ou mais cesáreas e as apresentações não cefálicas, uma vez que estes dois últimos fatores são considerados indicação absoluta de cesariana nesta instituição.

A cesariana foi a variável dependente, sendo dicotomizada em presente (caso) ou ausente (parto normal ou fórcipe - controle). As variáveis independentes foram: idade materna, primiparidade, idade gestacional, assistência pré-natal, antecedente de cesárea, antecedente de parto normal, ruptura prematura das membranas, gemelaridade, sindrome hemorrágica, síndrome hipertensiva, diabete melito e oligoâmnio.

A idade materna foi analisada, inicialmente, como variável contínua, para, a seguir, ser categorizada em adolescente, cuja idade era até 19 anos; adulta jovem, com idade entre 20-34 anos; 
adulta, quando maior ou igual a 35 anos. Primiparidade foi dicotomizada em ausente, quando a paciente nunca houvera parido antes (exceto o parto em questão) e presente, quando da presença de partos anteriores. A idade gestacional foi avaliada em semanas completas a contar da data última menstruação e, na ausência dessa informação, pela regra de McDonald ou pela ultra-sonografia. Foi categorizada em pré-termo, quando a idade gestacional estava entre a $28^{\mathrm{a}}$ e a $36^{\mathrm{a}}$ semana; a termo, com a idade gestacional entre a $37^{\mathrm{a}}$ e a $41^{\mathrm{a}}$ semana, ou pós-termo, quando igual ou maior que a $42^{a}$ semana. A assistência pré-natal foi categorizada como completa (número de consultas igual ou maior que seis) ou incompleta (número de consultas menor que seis).

$\mathrm{O}$ antecedente de cesárea foi caracterizado como a referência em prontuário de passado de cesariana. Antecedente de parto normal foi caracterizado como a referência de passado de parto vaginal. A ruptura prematura de membranas foi caracterizada como a referência de amniorrexe prematura anterior ao início de trabalho de parto, com diagnóstico firmado em base na história e exame clínico.

A gemelaridade foi caracterizada como a referência de gestação múltipla no prontuário médico. Síndrome hemorrágica referiu-se ao diagnóstico de descolamento prematuro da placenta normalmente inserida, placenta prévia, ruptura do seio marginal ou ruptura de vasa prévia. Sindrome hipertensiva foi caracterizada como a referência de pressão arterial sistólica maior ou igual a $140 \mathrm{mmHg}$ ou pressão diastólica maior ou igual a $90 \mathrm{mmHg}$. Diabete melito foi caracterizada como a referência em prontuário de diabete clínico ou gestacional. Oligoâmnio foi caracterizado como a referência de ultra-sonografia com indice de líquido amniótico inferior a $8 \mathrm{~cm}$, na ausência de associação com ruptura prematura das membranas.

Os dados foram obtidos no Serviço de Arquivo Médico e Estatística a partir do registro de admissão e alta e dos prontuários médicos. Do mês de novembro de 2001 até junho de 2002, a pesquisadora procedeu à análise dos registros das variáveis desse estudo, constantes dos prontuários neonatais e obstétricos dos indivíduos da amostra. Constatando obediência aos critérios de inclusão e exclusão, preencheram-se os campos da ficha de dados construída exclusivamente para esse estudo. O tamanho amostral inicial igualou-se a 4.285 pacientes após aplicação dos critérios de inclusão e exclusão.

As fichas de dados numeradas seqüencialmente compuseram o banco de dados construído e analisado por meio do programa Epi-Info, versão 6.04d, de 14 de junho de 2001, do Centers for Disease Control \& Prevention. Finda a avaliação da coerência dos dados das pacientes selecionadas na primeira fase, foram excluídas 659 pacientes $(15,4 \%)$. O tamanho amostral final foi de 3.626 pacientes, ou seja, $84,6 \%$ das pacientes elegiveis.

Para análise estatística dos dados, utilizouse o programa Epi-Info 2002, revisão 1, de novembro de 2002, do Centers for Disease Control \& Prevention, nas subrotinas Analysis-Statistics e Analysis-Advanced Statistics, Logistic Regression ${ }^{13}$.

A análise constou de três etapas: univariada, bivariada e multivariada. A análise univariada consistiu na análise estatística descritiva das variáveis de caracterização amostral, independentes e dependente, determinando-se as razões freqüenciais de dicotomização das variáveis. Considerado o critério de associação biológica entre as variáveis independentes e a dependente, procedeu-se à análise bivariada, testando a significância estatística com os testes de $\chi^{2}$ e $t$ de Student, ao nível de 0,05. A relação causa-efeito entre cada variável independente e a via de parto foi calculada pela razão de chance (odds ratio - OR), com intervalo de confiança em nível de $95 \%$.

$\mathrm{Na}$ análise multivariada adotou-se a regressão logística, cujo objetivo é produzir uma equação matemática que relacione a probabilidade de ocorrência de cesárea, decorrente da ação de mais de uma variável independente, que atua como fator determinante (fator de risco) ${ }^{14}$, cujas premissas foram: dicotomização da variável cesárea em ausente e presente, existência de relação biológica entre as variáveis independentes estudadas e a ocorrência de cesariana e emprego da função logística epidemiológica para relacionar a variável cesárea às variáveis independentes.

Identificadas as variáveis estatisticamente significativas, em nível de 0,05, na análise bivariada, procedeu-se à crítica para determinar a necessidade de dicotomizar variáveis independentes quantitativas, comparando-se modelos multivariados diferentes.

Identificado o modelo ideal, avaliou-se a multilinearidade, para análise da possibilidade de erro de estimativa de coeficientes. Foram aplicados os testes diagnósticos do modelo final, estimada a probabilidade de cada caso e determinada a probabilidade de acerto da indicação de cesárea, para avaliar a eficiência do serviço. O presente estudo foi aprovado pelo Comitê de Ética em Pesquisa do CISAM. 


\section{Resultados}

Das 3.626 mulheres estudadas, 990 foram submetidas a cesariana $(27,3 \%)$ e 2.636 tiveram parto normal $(72,7 \%)$. Estas pacientes tinham idade variando entre 12 e 48 anos, com média de $23,0 \pm 5,9$ anos e moda de 19 anos. Havia 1.179 $(32,5 \%)$ mulheres adolescentes, 2.268 (62,5\%) adultas jovens, na faixa etária entre 20 e 34 anos, e 179 (5\%) adultas, com idades iguais ou superiores a 35 anos.

Os resultados da associação entre ocorrência de cesárea e fatores de risco foram resumidos na Tabela 1, segundo razões de ocorrência. Os grupos etários e o tipo de gestação pré-termo não mostraram associação com o tipo de parto e não se configuraram como fatores de risco para cesárea. Pela análise bivariada foi observado que primiparidade, gravidez pós-termo, assistência pré-natal incompleta, antecedente de cesárea, ruptura prematura de membranas, gemelaridade, oligoâmnio, sindrome hipertensiva e sindrome hemorrágica constituíram fatores de risco para cesárea, ao passo que antecedente de parto vaginal foi identificado como fator de proteção para cesárea. As diferentes faixas etárias não estiveram significativamente associadas à cesariana.

Tabela 1 - Razão de chances das variáveis categóricas para cesariana.

\begin{tabular}{|c|c|c|c|c|c|c|c|c|}
\hline \multirow[t]{3}{*}{ Variável } & \multicolumn{4}{|c|}{ Pacientes } & \multirow[t]{3}{*}{ OR } & \multirow[t]{3}{*}{ IC $95 \%$} & \multirow[t]{3}{*}{$\chi^{2}$} & \multirow[t]{3}{*}{$\mathrm{p}$} \\
\hline & \multicolumn{2}{|c|}{ caso } & \multicolumn{2}{|c|}{ controle } & & & & \\
\hline & $\mathrm{n}$ & $\%$ & $\mathrm{n}$ & $\%$ & & & & \\
\hline Adolescente & 319 & 32,2 & 860 & 32,6 & 1,0 & $0,8-1,1$ & 0,0 & 0,82 \\
\hline $20-34$ anos & 611 & 61,7 & 1657 & 62,9 & 0,9 & $0,8-1,1$ & 0,4 & 0,53 \\
\hline$\geq 35$ anos & 60 & 6,1 & 119 & 4,5 & 1,4 & $1,0-1,9$ & 3,7 & 0,05 \\
\hline Primiparidade & 599 & 60,5 & 1125 & 42,7 & 2,0 & $1,8-2,4$ & 91,2 & $<0,001$ \\
\hline Gestação pré-termo & 173 & 17,5 & 490 & 18,6 & 0,9 & $0,8-1,1$ & 0,6 & 0,45 \\
\hline Gestação termo & 690 & 69,7 & 1935 & 73,4 & 0,8 & $0,7-1,0$ & 4,9 & 0,03 \\
\hline Gestação pós-termo & 127 & 12,8 & 211 & 8,0 & 1,7 & $1,3-2,1$ & 19,8 & $<0,001$ \\
\hline Assistência pré-natal incompleta & 529 & 56,8 & 1159 & 46,8 & 1,5 & $1,3-1,7$ & 26,9 & $<0,001$ \\
\hline Antecedente de cesárea & 242 & 24,4 & 136 & 5,2 & 5,9 & $4,7-7,4$ & 286,6 & $<0,001$ \\
\hline Antecedente de parto vaginal & 102 & 11,9 & 657 & 37,4 & 0,2 & $0,2-0,3$ & 182,0 & $<0,001$ \\
\hline Ruptura prematura de membranas & 162 & 16,4 & 334 & 12,7 & 1,3 & $1,1-1,6$ & 8,3 & $<0,001$ \\
\hline Gemelaridade & 25 & 2,5 & 10 & 0,4 & 6,8 & $3,3-14,2$ & 34,7 & $<0,001$ \\
\hline Oligoâmnio & 102 & 10,3 & 26 & 1,0 & 11,5 & $7,4-17,9$ & 183,4 & $<0,001$ \\
\hline Síndrome hipertensiva & 397 & 40,1 & 404 & 15,3 & 3,7 & $3,1-4,4$ & 256,7 & $<0,001$ \\
\hline Diabete melito & 17 & 1,7 & 6 & 0,2 & 7,7 & $3,0-19,5$ & 25,3 & $<0,001$ \\
\hline Síndrome hemorrágica & 35 & 3,5 & 2 & 0,1 & 48,3 & $11,6-201,1$ & 85,3 & $<0,001$ \\
\hline
\end{tabular}

OR = odds ratio; IC95\% = intervalo de confiança a $95 \%$.

Por meio da análise multivariada, o presente estudo permitiu mostrar que sindrome hemorrágica, oligoâmnio, diabete melito, gemelaridade, antecedente de cesárea, síndrome hipertensiva, gestação pós-termo, ruptura prematura de membranas e assistência pré-natal incompleta constituíram fatores de risco para cesárea, nessa ordem de influência (Tabela 2).

Posteriormente, calculou-se a probabilidade de cesárea basal para a amostra, ou seja, na ausência do todos os fatores de risco em estudo. Verificou-se que o risco basal de cesárea do presente modelo foi de $15,2 \%$, probabilidade decorrente, possivelmente, de outros fatores de risco não incluídos no presente estudo.

A Tabela 3 mostra a alteração da probabilidade de cesariana quando se acrescenta algum dos fatores de risco no modelo final. As três faixas etárias estudadas tiveram impactos distintos como fatores de risco para cesárea, de tal forma que idade igual ou superior a 35 anos acresceu $21,6 \%$ ao risco basal de cesárea e idade entre 20 e 34 anos aumentou em 4,3\% esse risco. A síndrome hemorrágica foi considerada o maior fator de risco, acrescendo a probabilidade em $78,7 \%$. O antecedente de parto vaginal e a idade inferior a 20 
Tabela 2 - Modelo preditivo final após eliminação das variáveis não significativas (adolescente, primiparidade, gestações pré-termo e termo).

\begin{tabular}{lccc}
\hline Variável & $\begin{array}{c}\text { Estimativa } \\
\text { do parâmetro }\end{array}$ & OR & IC 95\% \\
\hline Intercepto & $-1,7$ & & \\
$20-34$ anos & 0,3 & 1,3 & $1,1-5,6$ \\
$\geq 35$ anos & 1,2 & 3,2 & $1,9-5,6$ \\
Gestação pós-termo & 0,5 & 1,7 & $1,2-2,4$ \\
Assistência pré-natal incompleta & 0,3 & 1,3 & $1,0-1,6$ \\
Antecedente de cesárea & 1,8 & 6,0 & $4,6-7,9$ \\
Antecedente de parto vaginal & $-1,6$ & 0,2 & $0,2-0,3$ \\
Ruptura prematura de membranas & 0,5 & 1,6 & $1,2-2,1$ \\
Gemelaridade & 1,8 & 6,2 & $2,3-17,3$ \\
Oligoâmnio & 2,6 & 13,2 & $7,6-22,8$ \\
Síndrome hipertensiva & 1,3 & 3,6 & $2,9-4,5$ \\
Diabete melito & 2,5 & 12,6 & $3,3-47,8$ \\
Síndrome hemorrágica & 4,4 & 85,6 & $18,9-387,3$
\end{tabular}

Nota: a categoria de referência é a ausência do fator. OR = odds ratio; IC95\% = intervalo de confiança a $95 \%$.

$2 \log L$ (intercepto e variáveis) $=2400,7061, \chi^{2}=672,06$, g.l. $=12, p=0,00$

Razão de verossimilhança $=738,32$, g.l. $=12, p=0,00$

Tabela 3 - Alteração da probabilidade de cesárea decorrente da presença de cada um dos fatores de risco.

\begin{tabular}{lcc}
\hline Fatores de risco & Estimativas & $\begin{array}{c}\text { Alteração da } \\
\text { probabilidade } \\
\text { de cesárea (\%) }\end{array}$ \\
\hline Idade entre 20 e 34 anos & 0,3 & $+4,3$ \\
Idade $\geq 35$ anos & 1,2 & $+21,6$ \\
Presença de gestação pós-termo & 0,5 & $+8,3$ \\
Assistência pré-natal incompleta & 0,3 & $+11,3$ \\
Antecedente de cesárea & 1,8 & $+36,6$ \\
Antecedente de parto vaginal & $-1,6$ & $-11,6$ \\
Ruptura prematura de membranas & 0,5 & $+7,4$ \\
Presença de gemelaridade & 1,8 & $+37,6$ \\
Presença de oligoâmnio & 2,6 & $+55,0$ \\
Presença de síndrome hipertensiva & 1,3 & $+24,9$ \\
Presença de diabete melito & 2,5 & $+54,1$ \\
Presença de síndrome hemorrágica & 4,4 & $+78,7$ \\
\hline
\end{tabular}

anos se constituíram em fator de proteção para cesárea.

Aplicando o modelo logístico na amostra, determinou-se a associação entre a ausência dos fatores de risco estudados, ou seja, com probabilidade de cesárea inferior a $15,2 \%$, ou presença de um ou mais e a via de parto, como forma de avaliar a eficiência do serviço (Tabela 4).
Tabela 4 - Distribuição da presença de fator de risco avaliada pela probabilidade do modelo logístico e via de parto.

\begin{tabular}{lcccccc}
\hline Presença de fator de risco & \multicolumn{4}{c}{ Via de parto } & \multicolumn{2}{c}{ Total } \\
para cesárea, avaliada pelo & \multicolumn{2}{c}{ Cesárea } & \multicolumn{2}{c}{ Vaginal } & & \\
modelo logístico $(\mathbf{p}>\mathbf{1 5 , 2} \%)$ & $\mathbf{n}$ & $\%$ & $\mathbf{n}$ & $\%$ & $\mathbf{n}$ & $\%$ \\
\hline Nenhum & 133 & 13,4 & 1.414 & 53,6 & 1.547 & 42,7 \\
Um ou mais & 857 & 86,6 & 1.222 & 46,4 & 2.079 & 57,3 \\
Total & 990 & 100,0 & 2.636 & 100,0 & 3.626 & 100,0 \\
\hline
\end{tabular}

A Tabela 4 revela que $857(86,6 \%)$ gestantes foram submetidas à cesárea em presença de um ou mais fatores de risco; 133 (13,4\%) gestantes foram submetidas à cesárea em ausência de qualquer dos fatores de risco estudados (probabilidade inferior a $15,2 \%$ de indicação de cesárea pela regressão logistica); 1.414 (53,6\%) gestantes foram submetidas a parto vaginal sem qualquer fator de risco para cesárea, segundo o modelo logístico, e $1.222(46,4 \%)$ gestantes tiveram parto vaginal em presença de um ou mais fatores de risco para cesárea.

\section{Discussão}

Uma análise multivariada deve partir da existência de um problema comprovado, tal como se configura a elevada incidência de cesariana, embora tenha variado ao longo do tempo. Assim, nos Estados Unidos, de 1978 a 1986, variou de 14 a 28,8\% ${ }^{1}$, taxa que foi classificada como alta, quando comparada a outros países da Europa e do Pacífico, ao longo de 13 anos. No mesmo período, o crescimento do número de cesáreas na Europa foi bem menor ${ }^{11}$.

De modo análogo, a incidência de cesárea tem crescido no Brasil, tanto assim que há 30 anos, foi o país considerado o campeão de cesárea. Ao longo dos últimos cinco anos, no entanto, essa incidência tem declinado, sendo sua incidência de $26,4 \%$ em $2003^{15}$.

O mesmo fenômeno observou-se no CISAM, posto que, enquanto em 1997 a taxa alcançava os $28,5 \%{ }^{16}$, no presente estudo foi de $27,3 \%$, excluídas as apresentações pélvicas e a iteratividade. Embora a redução de 1,2\% entre 1997 e 2002 possa ser considerada pequena para um período de três anos, reflete as ações de saúde voltadas ao incentivo do parto vaginal, que incluem o treinamento de enfermagem obstétrica e a conscientização da população feminina para o parto humanizado.

O questionamento da alta incidência de cesárea fez com que fossem estudadas as condições biológicas da gestante que pudessem atuar como fatores de risco para essa via de parto. 
Embora na análise bivariada a idade materna a partir de 20 anos não tenha se comportado como fator de risco, a literatura refere a associação entre idades iguais ou superiores a 28 anos com maior freqüência de cesárea por intercorrências clínicas e maus antecedentes obstétricos ${ }^{5}$. $\mathrm{Na}$ análise multivariada do presente estudo, a idade maior que 20 anos foi o fator de risco para cesárea, no entanto pode-se aventar a possibilidade de o risco dever-se aos mesmos fatores.

Quanto à paridade, o resultado da análise bivariada permitiu detectar que as primíparas tiveram risco 2 vezes maior que as multíparas de serem submetidas a cesárea, portanto, inferior ao referido por Peleg et al. ${ }^{17}$.

$\mathrm{Na}$ análise multivariada, a primiparidade não se comportou como fator de risco para cesárea, tendo sido abandonada no processo de determinação do modelo ideal, por não apresentar significância estatística.

As mulheres com gestação pós-termo apresentaram chance aproximadamente $70 \%$ maior de serem submetidas à cesárea, na análise bivariada, dado corroborado por Matijeviae ${ }^{10}$ e por Alexander et al. ${ }^{18}$.

$\mathrm{Na}$ análise multivariada, o fator gestação pós-termo comportou-se como fator de risco para cesárea, ao passo que as gestações pré-termo e a termo não tiveram significância estatística.

Em relação ao número de consultas de prénatal, tanto na análise bivariada quanto na multivariada, este fator foi analisado como variável categórica, visto ser consenso do Ministério da Saúde que um total de seis ou mais consultas caracteriza assistência pré-natal completa. Em ambos os grupos, o número de consultas foi inferior ao indicado. De fato, para mulheres que foram submetidas à cesariana, a assistência incompleta atuou como fator de risco, tanto na análise bivariada quanto na multivariada, embora nesta tenha perdido importância, quando comparada ao valor de odds ratio da análise bivariada. Embora o número médio de consultas do grupo caso tenha se igualado a seis, pode-se supor que não tenha havido acompanhamento obstétrico adequado das possiveis intercorrências que surgiram durante a gravidez, fazendo com que essas gestantes chegassem ao parto em condições que indicaram cesariana.

A diferença entre os resultados da presente pesquisa e os obtidos por Trujillo Hernández et al. ${ }^{19}$, que constataram não ser o reduzido número de consultas fator de risco para cesárea, pode ser justificada pelo tipo de locais de estudo, pois enquanto a maternidade Professor Monteiro de Morais é pública, o serviço desses autores é privado, portanto, tem clientela com características sociais distintas.
Dentre as mulheres submetidas à cesárea, esse episódio único anterior se constituiu no quinto fator de risco de maior peso, em ambas as análises, bivariada e multivariada. Este resultado foi semelhante aos de outros estudos tipo casocontrole $^{17,19}$. Por outro lado, o antecedente de parto normal comportou-se como fator de proteção para cesariana, semelhantemente aos dados obtidos por Cunha et al. ${ }^{5}$.

Para a variável ruptura prematura de membranas, nossos resultados demonstraram que as pacientes com esta intercorrência tiveram 1,3 vezes mais chance de ser submetida à cesárea, a qual se elevou para 1,6 vezes, na análise multivariada. Estes dados se assemelham ao estudo de BenHaroush et al. ${ }^{20}$ e diferem do estudo de Hannah et al. ${ }^{21}$, o que sugere a existência de fatores que possam estar contribuindo para a associação entre ruptura prematura das membranas e cesárea na maternidade Professor Monteiro de Morais, por exemplo, a imaturidade do colo uterino. Este fato é corroborado pela associação entre ruptura prematura de membranas e primiparidade, uma vez que dentre as gestantes que apresentaram essa intercorrência, $57,9 \%$ eram primíparas, ao passo que $42,1 \%$, eram multíparas.

Os estudos abordando gemelaridade e via de parto evidenciaram maior risco de cesárea para o segundo gemelar ${ }^{22}$, o que dificulta a comparação com nossos resultados.

Quanto à variável oligoâmnio, a maioria dos estudos ${ }^{23,24}$ consideraram-no como fator de proteção para cesárea, diferentemente dos nossos resultados, que detectou chance 13 vezes maior de ser submetida à cesárea após ajuste das variáveis de confundimento. Por outro lado, por tratar-se de estudo retrospectivo, não foi possivel identificar as pacientes que tiveram o parto induzido por falta de registro nos prontuários. Este dado pode justificar o alto risco de cesárea nestas pacientes.

No presente estudo, sindrome hipertensiva ocupou o sexto lugar em influência sobre a ocorrência de cesárea, em ambas análises bivariada e multivariada. A comparação dos nossos dados com o da literatura ${ }^{5}$ ficou limitada, uma vez que não foi possivel categorizar esta variável quanto à forma clínica, por falta de detalhamento desta intercorrência no prontuário médico.

O diabete melito também se comportou como fator de risco para cesárea. Ehrenberg et al. ${ }^{25}$ observaram diabete na gestação na forma pré-gestacional constitui risco 3 vezes maior para esta tocurgia.

Por último, a sindrome hemorrágica foi o fator de risco mais elevado na análise bivariada e multivariada, atingindo valores de OR respectivamente de 48,3 e 85,6 , podendo-se aventar a possi- 
bilidade de constituir fator de indicação para cesárea. Nossos dados foram semelhantes aos obtidos por Cunha et al. ${ }^{5}$.

Segundo Pértega Diaz e Pita Fernández ${ }^{26}$, na maioria dos casos em que se pretende predizer uma resposta em função de um conjunto mais amplo de variáveis, é necessário considerar um modelo de regressão linear múltipla como extensão da reta de regressão que permite a inclusão de um número maior de variáveis.

Entretanto, na regressão linear múltipla, a variável resposta é numérica contínua. Diante de trabalho clínico em que se avaliam os efeitos de várias variáveis explanatórias sobre uma variável binária, no caso, a cesariana, o método de análise adequado é a regressão logística ${ }^{14}$.

Embora a amostra tenha sido obtida de serviço de referência, para o qual foram encaminhadas parturientes de outras unidades de saúde com menos recursos ou com insuficiência de leitos, a concentração dessas mulheres aumenta a probabilidade de ocorrência dos diversos fatores de risco, fazendo com que cada fator alcance um número suficiente para a análise estatística. Assim sendo, tal como referem Cunha et al. ${ }^{5}$ em estudo de coorte para análise de fatores de risco, o modelo logístico multivariado desse trabalho poderá não refletir a realidade de outros serviços de menor complexidade.

O presente estudo diferiu do modelo preditivo criado por Hin et al. ${ }^{12}$, que considerou variáveis intraparto, além das anteparto. Entretanto, assemelhou-se com o modelo criado por Cunha et al. ${ }^{5}$, apresentando valor preditivo positivo um pouco maior que este último, diferindo apenas pela exclusão do efeito cumulatico de cesáreas e as apresentações pélvicas, uma vez que estes fatores são considerados indicação para cesariana na instituição.

Para análise do risco basal de cesárea da ordem de $15,2 \%$, da ocorrência de $13,4 \%$ de cesáreas com baixa probabilidade de indicação pelo modelo logístico, assim como de $46,4 \%$ dos casos de parto vaginal que, teoricamente, teriam indicação de parto cirúrgico, deve-se considerar que o modelo calculado tomou por base exclusivamente fatores de risco anteparto, o que leva a supor que outros fatores intraparto possam ter atuado na decisão médica da via de parto.

O modelo permitiu prever corretamente $86,6 \%$ das cesáreas e $53,6 \%$ dos partos vaginais, podendo ser considerado bom e útil para a previsão da via de parto, para a avaliação da qualidade da assistência e para o planejamento dessa assistência, todavia, não constitui modelo acabado.

$O$ fato de o modelo logístico não ser linear pode representar alguma dificuldade de utilização prática, mesmo considerando que a equação que leva ao cálculo da probabilidade de cesárea é relativamente simples. Poder-se-á criar uma tabela de conversão de presença de fatores de risco em probabilidade de cesárea, com base em modelos desse tipo. O modelo logístico multivariado deve ser alvo de estudos mais detalhados, neste e em outros serviços, para contribuir para a redução da incidência de cesáreas, preservando o binômio materno-fetal.

Concluindo, o modelo preditivo final de cesárea permitiu identificar risco basal igual a $15,2 \%$, na ausência dos fatores de risco estudados. Essa probabilidade se eleva na presença de fatores anteparto de risco para cesárea, respectivamente em: 36,6\% na presença de antecedente de cesárea, $11,3 \%$ na assistência pré-natal incompleta, $24,9 \%$ na presença de síndrome hipertensiva, $54,1 \%$ na presença de diabete melito, $37,6 \%$ na gemelaridade, $8,3 \%$ na gestação pós-termo, $78,7 \%$ na presença de síndrome hemorrágica, $4,3 \%$ quando a idade materna estiver entre 20 e 34 anos ou $21,6 \%$ quando igual ou superior a 35 anos, 55,0\% na presença de oligoâmnio e $7,4 \%$ na presença de ruptura prematura de membranas.

Foi caracterizado como fator anteparto de proteção para operação cesariana o antecedente de parto vaginal, que reduz o risco de cesárea em 11,6\%.

\section{Referências}

1. Notzon FC, Placek PJ, Taffel SM. Comparisons of national cesarean-section rates. N Engl J Med. 1987;316(7):386-9.

2. Belizán JM, Berger E, Garrulity G. Epidemic cesarean section rates in Latin America. Acta Obstet Gynecol Scand Suppl. 1997;167 (Pt 1) 15.

3. Ministério da Saúde. Secretaria de Políticas de Saúde. Área Técnica de Saúde da Mulher. Parto, aborto e puerpério: assistência humanizada à mulher. Brasília: Ministério da Saúde; 2001. p. 32-7.

4. Behague DP, Victora CG, Barros FC. Consumer demand for caesarean sections in Brazil: informed decision making, patient choice, or social inequality? A population based birth cohort study linking ethnographic and epidemiological methods. BMJ. 2002;324(7343):942-5.

5. Cunha AA, Portela MC, Amed AM, Camano L. Modelo preditivo para cesariana com uso de fatores de risco. Rev Bras Ginecol Obstet. 2002;24:21-8.

6. Bailit JL, Love TE, Mercer B. Rising cesarean rates: are patients sicker? Am J Obstet Gynecol. 2004;191(3):800-3. 
7. Rosen MG, Dickinson JC. Vaginal birth after cesarean: a meta-analysis of indicators for success. Obstet Gynecol. 1990;76(5 Pt 1):865-9.

8. Kozinszky Z, Orvos H, Zoboki T, Katona M, Wayda $\mathrm{K}$, Pal A, et al. Risk factors cesarean section of primiparous women aged over 35 years. Acta Obstet Gynecol Scand. 2002;81(4):313-16.

9. Seyb ST, Berka RJ, Socol ML, Dooley SL. Risk of cesarean delivery with elective induction of labor at term in nulliparous women. Obstet Gynecol. 1999;94(4):600-7.

10. Matijeviae R. Outcome os post-term pregnancy: a matched-pair case-control study. Croat Med J [serial on the Internet]. 1998 [cited 2001 Feb 11];39(4):[about 11 p.]. Available from: http:// www.cmj.hr/ 1998/3904/390411.htm

11. Guihard P, Blondel B. Trends in risk factors for caesarean sections in France between 1981 and 1995: lessons for reducing the rates in the future. BJOG. 2001;108(1):48-55.

12. Hin LY, Lau TK, Rogers M, Chang AM. Antepartum and intrapartum prediction of cesarean need: risk scoring in singleton pregnancies. Obstet Gynecol. 1997;90(2):183-6.

13. Centers for Disease Control and Prevention (CDC). Epi Info [computer program]. Version 6.04. Atlanta (GA): CDC; 2002.

14. Kleinbaum DG. Logistic regression: a self-learning text. New York: Springer-Verlag; 1994.

15. Ministério da Saúde. Taxas de parto normal e cesárea no Brasil. J Febrasgo. 2004;11(3):4-6.

16. Moraes Filho OB, Costa CFF, Cabral SALCS. Incidência e indicações de cesariana na Maternidade Monteiro de Morais. An Nord Ginecol Obstet. 1998;21(1):6-8.

17. Peleg D, Hannah MF, Hodnett ED, Foster GA, Willan AR, Farine D. Predictors of cesarean delivery after prelabor rupture of membranes at term. Obstet Gynecol. 1999;93(6):1031-5.
18. Alexander JM, McIntire DD, Levine KJ. Prolonged pregnancy: induction of labor and cesarean births. Obstet Gynecol. 2001;97(6):911-5.

19. Trujillo Hernández B, Tene Pérez CE, Rios Silva M. Factores de riesgo para cesárea: un enfoque epidemiológico. Obstet Gynecol Méx. 2000;68:306-11.

20.Ben-Haroush, Yogev Y, Glickman H, Bar J, Kaplan B, Hod M. Mode of delivery in pregnancies with premature rupture of membranes at or before term following induction of labor with vaginal prostaglandin E2. Am J Perinatol. 2004;21(5):263-8

21. Hannah ME, Hannah WJ, Hawson SA, Hodnett ED, Saigal S, Willan AR. Planned caesarean section versus planned vaginal birth for breech presentation at term: a randomised multicentre trial. Term Breech Trial Collaborative Group. Lancet. 2000; 356(9239):1375-83.

22. Suzuki S, Yoneyama Y, Sawa R, Takeuchi M, Shin $\mathrm{S}$, Araki T. Fetal position associated with an increased risk of cesarean delivery in nulliparous twin gestations. Acta Obstet Gynecol Scand. 2001; 80(3):273-4.

23. Rainford M, Adair R, Scialli AR, Ghidini A, Spong CY. Amniotic fluid index in the uncomplicated term pregnancy. Prediction of outcome. J Reprod Med. 2001;46(6):589-92.

24. Verroti C, Bedocchi L, Piantelli G, Cavallotti D, Fieni $\mathrm{S}$, Gramellini D. Amniotic fluid index versus largest vertical pocket in the prediction of perinatal outcome in post-term pregnancies. Acta Biomed Ateneo Parmense. 2004;75 Suppl 1:67-70.

25. Ehrenberg HM, Durnwald CP, Catalano P, Mercer BM. The influence of obesity and diabetes on the risk of cesarean delivery. Am J Obstet Gynecol. 2004;191(3):969-74.

26. Pértega Díaz S, Pita Fernández S. Metodologia de la investigación. Técnicas de regresión: regresión lineal múltiple. Cad Aten Primaria [serial en Internet]. 2000 [citado 2002 jul 22]; 7 :[about 4 p.]. Disponible en: http://www.fisterra.com/mbe/ investiga/regre_lineal_multi/regre_lineal_multi.htm 